\title{
Prevention of COPD Readmissions: A Work in Progress
}

Patients discharged following in-patient stays for exacerbations of COPD often require unplanned return visits to the emergency department and/or readmission to the hospital. Twenty percent of Medicare-eligible patients ( $\geq 65 \mathrm{y}$ old) admitted for COPD exacerbations have been reported to require readmission within $30 \mathrm{~d} .{ }^{1}$ Half of these readmissions were for respiratory-related disease, with $27 \%$ identified as having another exacerbation as the reason for readmission.

The Hospital Readmissions Reduction Program was enacted to reduce rising costs of health care for Medicare beneficiaries due to readmissions for pneumonia, congestive heart failure, and acute myocardial infarction, with COPD added in 2014. Under the Hospital Readmissions Reduction Program, hospitals exceeding expected readmission rates for these diagnoses are penalized with a $3 \%$ reduction in all Medicare reimbursements. Identification of uninsured patients and patients who are dual-eligible for Medicaid and Medicare as a higher risk for readmission following COPD exacerbations has put safety net hospitals caring for these patients at risk for financial penalties.

Fiscal concerns and recognition that specific interventions may reduce COPD exacerbations have accelerated progress in developing and validating comprehensive disease management programs to improve care and reduce readmissions. Additional patient risk factors for readmission following hospitalization for COPD exacerbations include patients with more severe baseline disease (eg, home oxygen use, hypercapnia, comorbidities such as congestive heart failure, systemic corticosteroid use, continued smoking, substance abuse) and/or poor access to health care (eg, no identifiable primary care provider, transportation and language barriers). The recognition of COPD as a chronic, inflammatory, systemic disease with multiple clinical phenotypes and comorbidities (eg, malnutrition, osteoporosis, congestive heart failure, sleep-disordered

Dr Derdak has disclosed no conflicts of interest.

The views expressed are those of the author and do not represent the official views or policy of the Department of Defense or its components.

Correspondence: Stephen Derdak DO, Pulmonary/Critical Care Medicine, San Antonio Military Medical Center, Lackland AFB, Texas 78236. E-mail: sderdak@mac.com.

DOI: $10.4187 /$ respcare 05350 breathing, depression) invites comparison with other chronic lung diseases, such as asthma and cystic fibrosis, and with non-pulmonary diseases, such as diabetes or congestive heart failure, in which comprehensive disease management approaches are utilized. A common denominator in these diseases is the generally incurable nature and chronic relapsing courses.

\section{See the Original Study on Page 1}

The goals of chronic COPD management include optimizing quality of life, preserving or improving functional status, addressing associated comorbidities, and minimizing exacerbations. ${ }^{2}$ This is achieved in COPD with a range of evidence-based pharmacologic and non-pharmacologic approaches as recently summarized in practice guidelines by international organizations. ${ }^{3,4}$ Key non-pharmacologic approaches include case management, patient education with written action plans, pulmonary rehabilitation (ideally started within $30 \mathrm{~d}$ of discharge), and smoking cessation programs. Development of an effective disease management team requires integration of multiple elements and may benefit from emerging communication and monitoring technology. Technological advances linking outpatients with health-care professionals (eg, respiratory therapists, nurses, dietitians, pharmacists, physicians) have progressed faster then validation studies demonstrating improved outcomes. Examples include direct observation of inhaler technique by video-audio links (eg, Facetime, Skype), tablet- and/or smart phone-based applications (eg, COPD Navigator), digital diaries for symptom tracking, home pulse oximetry, peak expiratory flow, and inspiratory capacity monitoring.

The ability to extend the reach of the hospital or clinicbased respiratory therapist to the patient's home after hospital discharge may have the potential to improve outcomes and quality of life. In this context, the study by Silver et $\mathrm{al}^{5}$ in this issue of RESPIRATORY CARE is especially relevant to the essential role of respiratory therapists in preventing readmissions following COPD exacerbation. These investigators randomized 428 subjects at high risk for readmission to usual pre-discharge care versus a comprehensive respiratory therapy disease management program conducted by specially trained respiratory therapist case managers. ${ }^{6}$ Study enrollment was restricted to sub- 


\section{EDITORIALS}

jects $\leq 65$ y old, since an existing Medicare readmission prevention program was already in place at the authors' institution. Study eligibility required prespecified risk factors for COPD exacerbation readmissions (eg, previous emergency department visits, hospitalizations, use of systemic corticosteroids within the previous year, or requirement for home oxygen). The respiratory therapy disease management protocol incorporated pre-discharge subject education (including proper inhaler and guideline-appropriate medication use), coordination of out-patient clinic appointments, pulmonary rehabilitation, and smoking cessation interventions and included 5 structured follow-up telephone calls by the respiratory therapist case manager.

Key findings of Kollef et $\mathrm{a}^{5}$ were that subjects receiving respiratory therapy disease management experienced significantly fewer readmissions for COPD exacerbations ( $20 \%$ vs $28 \%$ ), shorter duration of hospitalization if readmission was required ( $5 \mathrm{~d}$ vs $8 \mathrm{~d}$ ), and fewer total inpatient and ICU days over 6 months of follow-up. Mortality was similar (and low) between both groups (1.4\% vs $0.9 \%$ ). Interestingly, the reduction in readmissions for COPD exacerbations in the respiratory therapy disease management study group occurred despite significantly more subjects in this group being dual Medicare-Medicaid eligible and probably having COPD-asthma overlap, both factors associated with a higher risk of readmissions in Medicare beneficiaries. ${ }^{2}$ All cause readmissions and nonCOPD-related emergency department visits did not differ between study groups. Strengths of this respiratory therapy disease management discharge program included the relatively low cost (eg, respiratory therapist case-management, subject teaching, written action plan, telephone follow-up), minimal technology required (telephone), and applicability to a diverse population of subjects with COPD exacerbation.

In contrast, technology-based approaches described above, although promising, have not yet consistently demonstrated reduction in COPD exacerbation readmission rates. ${ }^{8,9}$ The study by Silver et $\mathrm{al}^{5}$ adds to the growing evidence base supporting use of a structured, pre-discharge comprehensive disease-management approach for high-risk patients admitted for COPD exacerbations. Respiratory therapy departments may use this study as an example of how to develop a hospital-specific program to prevent COPD readmissions, improve patient care, and potentially reduce costs. Whether incorporating emerging technologies (eg, video-patient conferencing, web-based symptom diaries, tablet applications, digital transmission of home pulse oximetry, peak expiratory flow, inspiratory capacity, portable spirometry) into respiratory therapist-directed COPD disease management programs will provide added benefit in reducing COPD readmissions will require future research. As with many efforts to improve patient care for chronic diseases, respiratory therapist-directed disease management programs for COPD might be viewed as a work in progress, subject to revision as new preventive therapies, patient education techniques, and monitoring technologies are validated.

Stephen Derdak DO Pulmonary/Critical Care Medicine San Antonio Military Medical Center Lackland Air Force Base, Texas

\section{REFERENCES}

1. Shah T, Churpek MM, Coca Perraillon M, Konetzka RT. Understanding why patients with COPD get readmitted: a large national study to delineate the Medicare population for the readmissions penalty expansion. Chest 2015;147(5):1219-1226.

2. Lange P, Çolak Y, Ingebrigtsen TS, Vestbo J, Marott JL. Long-term prognosis of asthma, chronic obstructive pulmonary disease, and asthma-chronic obstructive pulmonary disease overlap in the Copenhagen City Heart study: a prospective population-based analysis. Lancet Respir Med 2016;4(6):454-462.

3. Global Initiative for Chronic Obstructive Lung Disease (GOLD). Global strategy for the diagnosis, management and prevention of COPD, 2016. http://goldcopd.org/global-strategy-diagnosis-management-preventioncopd-2016/. Accessed October 14, 2016.

4. Criner GJ, Bourbeau J, Diekemper RL, Ouellette DR, Goodridge D, Hernandez P, et al. Prevention of acute exacerbations of COPD American College of Chest Physicians and Canadian Thoracic Society Guideline. Chest 2015;147(4):894-942.

5. Silver PC, Kollef MH, Clinkscale D, Watts P, Kidder R, Eads B, et al. A respiratory therapist disease management program for patients hospitalized with COPD. Respir Care 2017;62(1):1-9.

6. American Association for Respiratory Care. COPD educator course. http://www.aarc.org/education/online-courses/copd-educator-course/. Accessed October 13, 2016.

7. Marchetti N, Criner GJ, Albert RK. Preventing acute exacerbations and hospital admissions in COPD. Chest 2013;143:1444-1454.

8. McDowell JE, McClean S, FitzGibbon F, Tate S. A randomised clinical trial of the effectiveness of home-based health care with telemonitoring in patients with COPD. J Telemed Telecare 2015;21(2):8087.

9. Pinnock H, Hanley J, McCloughan L, Todd A, Krishan A, Lewis S, et al. Effectiveness of telemonitoring integrated into existing clinical services on hospital admission for exacerbation of chronic obstructive pulmonary disease: researcher blind, multicentre, randomised controlled trial. BMJ 2013;347:f6070. 